\title{
A LH-DM Strategy Based Particle Swarm Optimization Algorithm
}

\author{
W. Liu \\ College of Telecommunications and Information Engineering \\ Nanjing University of Posts and Telecommunications \\ Nanjing, China
}

\begin{abstract}
Aiming at the premature problem of the Particle S warm Optimization (PSO), an improved algorithm based on dynamic mutation strategy named Lowdiversity and Highdiversity Dual Mutation Factors Particle Swarm Optimization (LH-DMPSO) was proposed. The dynamic mutation strategy enhanced particle diversity, avoid falling into local optimal position, simulation results based on the Benchmark functions shows that the improved algorithm exceeds the standard PSO and Gaussian Mutation Partide Swarm Optimization (GMPSO) on convergence and stability.
\end{abstract}

Keywords-premature convergence; dynamic mutation; particle diversity

\section{INTRODUCTION}

Particle Swarm Optimization (PSO) [1] is a swarm intelligent algorithm proposed by the social psychologist Dr Kennedy and the Electronic Engineer Dr Eberhart in 1995. Because of its simplicity, fast convergence speed and advantages of less adjustable parameters, PSO is widely used in nonlinear function planning [2], power system [3], path planning problem $[4]$ and so on. However, when solving complex nonlinear optimization problem, PSO is easy to fall into the premature convergence problem. In order to deal with this shortcoming, many solutions were proposed. A. Ratnaweera [5] has proposed a linear variation of the acceleration factor strategy, which the cognitive factor linear decline, while social factors linear increase during the search process. R. K. Ursem [6] has proposed a diversity guided strategy to control the swam moving. By measuring the diversity he let the swarm alternate between attraction and repulsion phases.

In this paper, we present an improved scheme to deal with the problem. we proposed a dynamic mutation strategy based on the average particle distance to maintain the swam diversity and so as to solve the premature issue. Tests on the selected four Benchmark functions show that our algorithm has better search performance than standard PSO and Gaussian mutation particle swarm optimization (GMPSO).

\section{PARTICLE SWARM OPTIMIZATION}

In the Particle Swarm Optimization, each particle can be viewed as a solution to the problem in the feasible space. All particles constantly adjust their status through tracking the global best position gbest and each particle's historical best position pbest $\mathrm{i}_{\mathrm{i}}$, so as to complete the search process.

\author{
J. Zhou \\ College of Telecommunications and Information Engineering \\ Nanjing University of Posts and Telecommunications \\ Nanjing, China
}

Suppose that the swam composed of M particles. Position vector of $i^{\prime}$ th particle : $x_{i}=\left(x_{i 1}, x_{i 2}, \ldots, x_{i D}\right), 1 \leq \mathrm{i} \leq \mathrm{M}$, velocity vector of i'th particle: $v_{i}=\left(v_{i 1}, v_{i 2}, \ldots, v_{i D}\right), 1 \leq \mathrm{i} \leq \mathrm{M}$, the i'th particle's

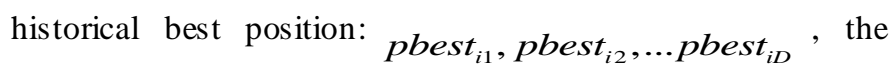
swarm's best position: $g$ best, $\mathrm{f}(\mathrm{x})$ is the fitness function, then the i-the particle's current historical best position determined by the following formula (1).

$$
\text { pbest }_{i}(\mathrm{k}+1)=\left\{\begin{array}{r}
\text { pbest }_{i}(\mathrm{k}) \text {, if } \mathrm{f}\left(\mathrm{x}_{\mathrm{i}}(\mathrm{k}+1)\right) \geq \mathrm{f}\left(\text { pbest }_{i}(\mathrm{k})\right) \\
x_{i}(\mathrm{k}+1), \text { if } \mathrm{f}\left(\mathrm{x}_{\mathrm{i}}(\mathrm{k}+1)\right)<\mathrm{f}\left(\text { pbest }_{i}(\mathrm{k})\right)
\end{array}\right.
$$

The swarm's best position gbest determined by the following formula (2).

$$
\text { gbest }=\min \left\{\mathrm{f}\left(\text { pbest }_{1}\right), \mathrm{f}\left(\text { pbest }_{2}\right), \mathrm{f}\left(\text { pbest }_{3}\right), \ldots, \mathrm{f}\left(\text { pbest }_{m}\right)\right\}
$$

At each step, all particles update their position and velocity vector, as in the formula (3) and (4).

$$
\begin{gathered}
\mathrm{v}_{\mathrm{id}}^{\mathrm{k}+1}=\mathrm{w}^{*} \mathrm{v}_{\mathrm{id}}^{\mathrm{k}}+\mathrm{r}_{1} * \mathrm{c}_{1} *\left(\text { pbest }_{\mathrm{id}}^{\mathrm{k}}-\mathrm{x}_{\mathrm{id}}^{\mathrm{k}}\right)+\mathrm{r}_{2} * \mathrm{c}_{2} *\left(\text { gbest }_{\mathrm{d}}^{\mathrm{k}}-\mathrm{x}_{\mathrm{id}}^{\mathrm{k}}\right) \\
\mathrm{x}_{\mathrm{id}}^{\mathrm{k}+1}=\mathrm{x}_{\mathrm{id}}^{\mathrm{k}}+\mathrm{v}_{\mathrm{id}}^{\mathrm{k}+1}
\end{gathered}
$$

where, $i$ is the $i^{\prime}$ th particle, $d$ is the dimension of search space, $\mathrm{k}$ is the number of iterations. ${ }_{r_{1}}, r_{2}$ are random numbers, usually $[0,1], c_{1}, c_{2}$ are acceleration factor, the parameter $\mathrm{w}$ is the inertia weight and controls the step magnitude. Once the particles are constraint by a local extreme value, the swarm falls into premature convergence problem.

\section{LH-DM PART ICLE SWARM OPTIMIZATION}

Reference [7] found that the premature problem was closely related to the population diversity, the population diversity will decline with the progress of the algorithm. In this paper, we first introduce the dual mutation factors [8] to tracing the best particle and the worst particle respectively ; then we propose a diversity-guided dynamic mutation to maintain the population diversity, and then avoid the premature problem.

\section{A. Average Particle Distance}

Reference [9] proposed to use average particle distance to measure the population diversity:

$$
\operatorname{diversity}(\mathrm{m})=\frac{1}{|m| *|L|} * \sum_{i=1}^{|m|} \sqrt{\sum_{j=1}^{D}\left(\mathrm{p}_{\mathrm{i} j}-\overline{p_{j}}\right)^{2}}, \overline{p_{j}}=\frac{1}{N} \sum_{i=1}^{m} \mathrm{p}_{\mathrm{i} j}
$$


where, $\mathrm{m}$ is the swarm, $|\mathrm{m}|$ is the swarm size, $|L|$ is the length of longest the diagonal in the search space, $\mathrm{D}$ is the dimension of the problem. Diversity (m) has measured the swarm average distance to the swarm center. it is independent of swarm size, the dimensionality of the problem as well as the search range in each dimension. Smaller diversity $(\mathrm{m})$ means the distribution of the particles is more concentrated and is more easy to fall into local optimal value, conversely can achieve a more dispersed distribution and avoid this problem by a large probability.

\section{B. Dual Mutation Factors}

Dual Mutation Factors are consist of the variables Ybest and Yworst. the best factor Ybest is used to trace the particle with best fitness value, accordingly, the worst factor Yworst is used to trace the particle with worst fitness value. In each iteration, we execute mutation according to formula (6) on the best particle which Ybest traced so that the best particle will be guided to search near current global best position and improve the accuracy of the result. Conduct mutation according to formula (7) on the worst particle which Yworst traced so that the worst particle can search a new area far from the current global best position and improve the Average Particle Distance (APD).

$$
\begin{gathered}
X=\text { Yworst }^{*}(1+0.618 \text { randn }) \\
X=\text { Ybest }^{*}(1+0.5 \text { randn })
\end{gathered}
$$

where randn is the random number generated according to the Gaussian probability distribution, i.e. $\mathrm{N}(0,1)$.

\section{LH-DM Strategy}

In this paper, we propose a dynamic mutation strategy according to the changes of the average particle distance. When diversity $(\mathrm{m})$ is lower than the preset Lowdiversity, execute formula (6); when diversity(m) is higher than the preset Highdiversity, execute formula (7).

Lowdiversity and Highdiversity Dual Mutation Factors (LH-DM) strategy:

$$
\begin{aligned}
& \text { if } \operatorname{diversity}(\mathrm{m})<\text { Lowdiversity } \\
& \text { execute formula (6); } \\
& \text { if } \operatorname{diversity}(m)>\text { Highdiversity } \\
& \text { execute formula (7); }
\end{aligned}
$$

which, Lowdiversity and Highdiversity are preset minimum and maximum threshold values according to the complexity of the fitness function,we refer to the reference [6] set Lowdiversity $=5.0 * 10^{-6}$, Highdiversity $=0.25$.

\section{D. $L H$-DMPSO}

The proposed algorithm description is presented below:

1. parameters setting, initialize each particle's position vector and velocity vector;

2. initialize each particle's pbest $\mathrm{i}_{\mathrm{i}}$ and the swarm's gbest according to formula (1) and formula (2) respectively, setting the best particle's position as Ybest, worst particle's position as Yworst;

3. calculate divsersity $(\mathrm{m})$ according to formula (5), execute mutation according to formula (6), formula (7);

4. update each particle's velocity vector according to formula (3);

5. update each particle's position vector according to formula
6. calculate each particle's fitness, then update pbest ${ }_{i}$, gbest, Ybest, Yworst;
7. Iteration from step 3 until reaching the final stop.

\section{SIMULATION RESULT S}

In order to test the performance of LH-DMPSO, we

\begin{tabular}{|c|c|c|c|}
\hline Punction & Expression & $\begin{array}{l}\text { Defined } \\
\text { field }\end{array}$ & $\begin{array}{r}\text { Optimal } \\
\text { solution }\end{array}$ \\
\hline Sphere & $f(x)=\sum_{i=1}^{n} x_{i}^{2}$ & {$[-100,100]$} & 0 \\
\hline Ackley & $f(x)=-20 \exp \left(-0.2 \sqrt{\frac{1}{n} \sum_{i=1}^{1} x_{i}^{*}}\right)-\exp \left(\frac{1}{n} \sum_{i=1}^{1} \cos 2 \pi x_{i}\right)+20+e$ & {$[-5,5]$} & 0 \\
\hline Gri ewangk & $f(x)=\sum_{i=1}^{i} \frac{x_{i}^{2}}{4000}-\prod_{i=1}^{i} \cos \left(\frac{x_{i}}{\sqrt{i}}\right)+1$ & {$[-600,600]$} & 0 \\
\hline Rastrigrin & $f(x)=\dot{\sum}\left[x_{i}:-10 \cos (2 \pi x)+10\right]$ & {$[5.12,5,12]$} & 0 \\
\hline
\end{tabular}
conduct simulations compared with the Standard particle swarm optimization (PSO) and Gaussian mutation particle swarm optimization (GMPSO) on four Benchmark functions.

\section{A. Benchmark Functions}

The LH-DMPSO has been tested on four different functions:

\section{B. Results and Discussion}

Parameter settings: Three algorithms parameter setting are basically the same. Population size $\mathrm{m}=50$, Dimension sizes: 10 , 20 and $30, w=1.412413$, The maximu $m$ number of generations $\mathrm{N}=5000$, The accelerating constants $\mathrm{c}_{1}=2$ and $\mathrm{c}_{2}=2$, target optimal fitness value is set to $1.0 * 10^{-5}$. Such running trials were repeated for each of the chosen function for 50 times. The experimental results are presented in Table 1 4. Dim is dimension of search space, Avebest is the average best fitness value of 50 tests. Aveiter is the average iterations when reach the target optimal fitness, '-' means search failure. Ras means algorithm success rate.

\section{TABLE I. THE RESULT S OFT HE TIRALS}

\begin{tabular}{|c|c|ccc|ccc|ccc|}
\hline \multirow{3}{*}{ Function } & & \multicolumn{3}{|c|}{ PSO } & \multicolumn{3}{c|}{ GMPSO } & \multicolumn{3}{c|}{ LH-DMPSO } \\
& Dim & Avebest & Aveiter & Ras & Avebest & Aveiter & Ras & Avebest & Aveiter & Ras \\
\hline \multirow{3}{*}{ Sphere } & 10 & $3.63 \times 10^{-7}$ & 423.72 & $50 / 50$ & $6.72 \times 10^{-11}$ & 217.23 & $50 / 50$ & 0 & 213.21 & $50 / 50$ \\
\cline { 2 - 10 } & 20 & 0.2231 & 637.24 & $0 / 50$ & $9.13 \times 10^{-9}$ & 353.11 & $50 / 50$ & $2.15 \times 10^{-11}$ & 335.43 & $50 / 50$ \\
\cline { 2 - 10 } & 30 & 1.0772 & - & $0 / 50$ & $2.32 \times 10^{-8}$ & 381.46 & $50 / 50$ & $3.47 \times 10^{-9}$ & 365.22 & $50 / 50$ \\
\hline
\end{tabular}

TABLE II.THE RESULT S OF THE TIRALS

\begin{tabular}{|l|c|ccc|ccc|ccc|}
\hline \multirow{3}{*}{ Function } & \multirow{3}{*}{ Dim } & Avebest & Aveiter & Ras & Avebest & Aveiter & Ras & Avebest & Aveiter & Ras \\
\hline \multirow{3}{*}{ Ackley } & 10 & 0.0053 & 467.21 & $50 / 50$ & $3.37 \times 10^{-6}$ & 286.21 & $49 / 50$ & $1.23 \times 10^{-11}$ & 245.72 & $50 / 50$ \\
\cline { 2 - 10 } & 20 & 0.2871 & - & $0 / 50$ & $9.12 \times 10^{-6}$ & 374.91 & $46 / 50$ & $5.33 \times 10^{-9}$ & 335.23 & $50 / 50$ \\
\cline { 2 - 10 } & 30 & 1.103 & - & $0 / 50$ & $1.03 \times 10^{-5}$ & 494.53 & $35 / 50$ & $7.86 \times 10^{-8}$ & 416.77 & $50 / 50$ \\
\hline
\end{tabular}

TABLE III.THE RESULT S OFT HE TIRALS

\begin{tabular}{|l|c|ccc|ccc|ccc|}
\hline \multirow{2}{*}{ Function } & & \multicolumn{3}{|c|}{ PSO } & \multicolumn{3}{c|}{ GMPSO } & \multicolumn{3}{c|}{ LH-DMPSO } \\
\hline \multirow{3}{*}{ Griewangk } & Avebest & Aveiter & Ras & Avebest & Aveiter & Ras & Avebest & Aveiter & Ras \\
\cline { 2 - 10 } & 10 & 0.1041 & - & $0 / 50$ & 0.0867 & 486.21 & $15 / 50$ & $1.01 \times 10^{-5}$ & 324.77 & $50 / 50$ \\
\cline { 2 - 10 } & 20 & 0.2035 & - & $-0 / 50$ & 0.1411 & 633.96 & $11 / 50$ & $0.97 \times 10^{-4}$ & 613.09 & $50 / 50$ \\
\cline { 2 - 10 } & 30 & 1.0261 & - & $-0 / 50$ & 0.9731 & - & $0 / 50$ & $9.21 \times 10^{-4}$ & 1103.11 & $38 / 50$ \\
\hline
\end{tabular}


TABLE IV.THE RESULT S OF THE TIRALS

\begin{tabular}{|l|c|ccc|ccc|ccc|}
\hline \multirow{3}{*}{ Function } & \multirow{2}{*}{ Dim } & Avebest & Aveiter & Ras & Avebest & Aveiter & Ras & Avebest & Aveiter & Ras \\
\hline \multirow{3}{*}{ Rastrigrin } & 10 & 6.3713 & - & $0 / 50$ & $5.07 \times 10^{-6}$ & 413.51 & $50 / 50$ & $7.63 \times 10^{-9}$ & 373.22 & $50 / 50$ \\
\cline { 2 - 10 } & 20 & 15.5246 & - & $0 / 50$ & $1.64 \times 10^{-4}$ & 583.73 & $37 / 50$ & $6.87 \times 10^{-7}$ & 516.41 & $50 / 50$ \\
\cline { 2 - 10 } & 30 & 54.7634 & - & $0 / 50$ & 0.0937 & - & $0 / 50$ & $0.17 \times 10^{-3}$ & 1469.15 & $31 / 50$ \\
\hline
\end{tabular}

From the results obtained, it can be observed that for the four benchmark minimization problems, compared to PSO and GMPSO, LH-DMPSO can achieve better search per-formance. For Sphere function, when the search space is 10 dimension, the average best fitness value algorithm can converge to 0 using LH-DMPSO, for the three multi peak functions Ackley, Griewangk and Rastrigrin, LH-DMPSO can obtain a smaller average best fitness value, means that the proposed algorithm can achieve good performance when solving high dimension, multi peak problems. Further more, analysis the average iterations of each table, LH-DMPSO is smaller than the standard PSO and GMPSO, means that the proposed algorithm can converge to the target value faster. Finally analysis the Ras in table 1 4, LH-DMPSO has the highest success rate, means a higher stability.

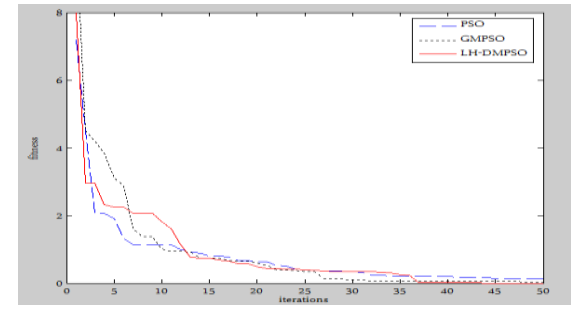

FIGURE I. M=50, DIM=20 RESULT FOR SPHERE FUNCTION

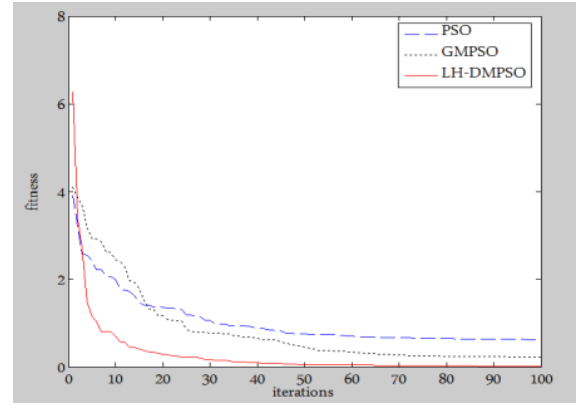

FIGURE II. M=50, DIM=30 RESULT FOR ACKLEY FUNCTION

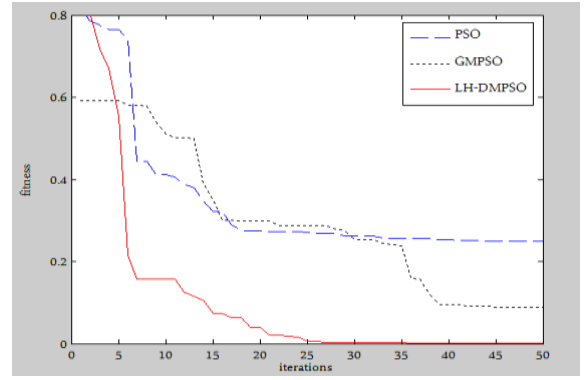

FIGURE III. M=50, DIM=10 RESULT FOR GRIEWANGK FUNCTION

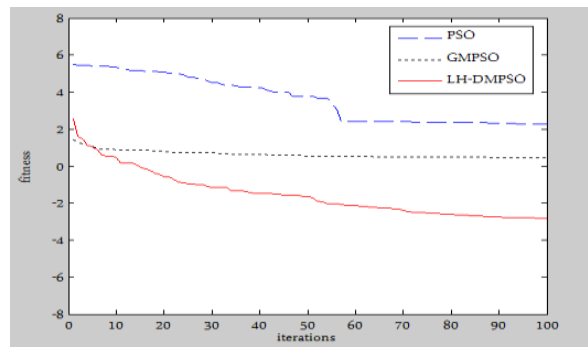

FIGURE IV. M=50, DIM=30 RESULT FOR RAST RIGRIN FUNCTION

Figure 1 4 shows a more intuitive comparison between the standard PSO, GMPSO and LH-DMPSO, it can be observed that for the four benchmark functions, the LH-DMPSO algorithm shows considerably better convergence than the standard PSO and GMPSO. The solid line represent for LHDMPSO can get a smaller fitness function value; While for Sphere function shown in Figure 1, the proposed algorithm performance improvement is not obvious, mainly because, for Sphere function, it is relatively easy to find its global best position, using standard PSO and GMPSO can also get satisfactory results. However, for Figure 2 4, LH-DMPSO performs significantly better, verifies that the proposed algorithm can achieve a more satisfactory performance when solving high-dimensional and multi-peak problems.

\section{CONCLUSIONS}

In this paper, a LH-DMPSO has been proposed to solve the PSO premature problem. By introducing dynamic mutation, we can maintain the population diversity at a satisfactory level, so as to avoid falling into local optimal. Finally, we conduct tests on four typical Benchmark functions, simulation results show that our proposed algorithm can achieve a lower average best fitness value, especially when solving high dimension and multi peak problems.

\section{ACKNOWLEDGEMENT S}

This work is Supported by National Natural Science Foundation of China (61271240) and Scientific Research Foundation of Nanjing University of Posts and Telecommunications (NY208018).

\section{REFERENCES}

[1] J. Kennedy, R. C. Eberhart, Particle swarm optimization, Proc. IEEE International Conference on Neural Networks, [C], Perth, Australia, 1942-1948, 1995.

[2] Y. Dong, J. Tang, B. Xu, D. Wang, An application of swarm optimization to nonlinear programming, Computer and Mathematics with Applications, [J], 49:1655-1668, 2005.

[3] C. W. Jiang, B. Etorre, A hybrid method of chaotic particle swarm optimization and linear interior for reactive power optimization, Mathematics and Computers in Simulation, [J], 68: 57-65, 2005.

[4] J. M. Xiao, J. J. Li, X. H. Wang, Improved Particle Swarm Optimization for vehicle routing problem.Computer integrated manufact uring system, [J], 11(4): 577-581, 2005.

[5] A. Ratnaweera, S. K. Halgamuge, H. C. Watson, Self-organizing hierarchical particle swarm optimizer with time-varying acceleration Coefficients. IEEE Transactions on Evolutionary Comput ation, [J], 8(3): 240-255, 2004.

[6] R. K. Ursem, Diversity-guided evolutionary algorithm, The 7th International Conference on Parallel Problem Solving from Nature, [C], LNCS2439. Berlin, Springer, 462-474, 2002.

[7] F. Liu, G. Z. Liu. Markov chain analysis and the convergence speed of 
genetic algorithms, Systems Engineering Journal, [J], 1998, 13(4): 79-

85.

[8] W.T. Xue, X. B. Wu, Z. L. Xu. An immune net work algorithm based on double mutation operators. Control and decision, [J], 2008, 23(12).

[9] R. A. Krohling, Gaussian swam: A novel particle swarm optimization algorithm, 2004 IEEE Conference on Cybernetics and Intelligent Systems, [C], Singapore, IEEE Inc, 372-376, 2004. 